\title{
Hepatic Arterial Infusion Chemotherapy for Patients with Huge Unresectable Hepatocellular Carcinoma
}

\author{
Wei-Lun Tsai ${ }^{1,2}$, Kwok-Hung Lai ${ }^{1,2}$, Huei-Lung Liang ${ }^{2,3}$, Ping-I Hsu ${ }^{1,2}$, Hoi-Hung Chan ${ }^{1,2}$, Wen-Chi Chen ${ }^{1,2}$, \\ Hsien-Chung $\mathrm{Yu}^{1,2}$, Feng-Woei Tsay ${ }^{1,2}$, Huay-Min Wang ${ }^{1,2}$, Hung-Chih Tsai ${ }^{4}$, Jin-Shiung Cheng ${ }^{1 *}$
}

1 Division of Gastroenterology, Department of Internal Medicine, Kaohsiung Veterans General Hospital, Kaohsiung, Taiwan, 2 School of Medicine, National Yang-Ming University, Taipei, Taiwan, 3 Department of Radiology, Kaohsiung Veterans General Hospital, Kaohsiung, Taiwan, 4 Department of Finance and Banking, College of Business and Management, Kun Shan University, Tainan, Taiwan

\begin{abstract}
Background and Aim: The optimal treatment for huge unresectable hepatocellular carcinoma (HCC) remains controversial. The outcome of transcatheter arterial chemoembolization (TACE) for patients huge unresectable HCC is generally poor and the survival benefit of TACE in these patients is unclear. The aim of the study is to compare the effect of hepatic arterial infusion chemotherapy (HAIC) versus symptomatic treatment in patients with huge unresectable HCC.

Methods: Since 2000 to 2005, patients with huge (size $>8 \mathrm{~cm}$ ) unresectable HCC were enrolled. Fifty-eight patients received HAIC and 44 patients received symptomatic treatment. In the HAIC group, each patient received 2.4+1.4 (range: 1-6) courses of HAIC. Baseline characteristics and survival were compared between the HAIC and symptomatic treatment groups.

Results: The HAIC group and the symptomatic treatment group were similar in baseline characteristics and tumor stages. The overall survival rates at one and two years were $29 \%$ and $14 \%$ in the HAIC group and $7 \%$ and $5 \%$ in the symptomatic treatment group, respectively. The patients in the HAIC group had significantly better overall survival than the symptomatic treatment group $(\mathrm{P}<0.001)$. Multivariate analysis revealed that HAIC was the significant factor associated with the overall survival (relative risk: $0.321,95 \%$ confidence interval: $0.200-0.515, \mathrm{P}<0.001$ ). None of the patients died due to immediate complications of HAIC.
\end{abstract}

Conclusions: HAIC is a safe procedure and provides better survival than symptomatic treatment in patients with huge unresectable HCC.

Citation: Tsai W-L, Lai K-H, Liang H-L, Hsu P-I, Chan H-H, et al. (2014) Hepatic Arterial Infusion Chemotherapy for Patients with Huge Unresectable Hepatocellular Carcinoma. PLoS ONE 9(5): e92784. doi:10.1371/journal.pone.0092784

Editor: Erica Villa, University of Modena \& Reggio Emilia, Italy

Received October 25, 2013; Accepted February 25, 2014; Published May 13, 2014

Copyright: ( $\odot 2014$ Tsai et al. This is an open-access article distributed under the terms of the Creative Commons Attribution License, which permits unrestricted use, distribution, and reproduction in any medium, provided the original author and source are credited.

Funding: The authors have no support or funding to report.

Competing Interests: The authors have declared that no competing interests exist.

*E-mail: rcheng@ms2.hinet.net

\section{Introduction}

Hepatocellular carcinoma (HCG) is the fifth most common cancer in the world and it is the $2^{\text {nd }}$ leading cause of cancer death in Taiwan [1,2]. Although high- risk patients are routinely screened in Taiwan, huge HCGs with size of more than $8 \mathrm{~cm}$ are occasionally seen [3]. Treating patients with huge HCG is difficult. Surgical resection is considered to be the standard curative therapy for HCC in patients with good liver reserve [4-8]. According to the study from our hospital, Mok et al. found that the advantage of hepatic resection in patients with huge $\mathrm{HCC}$ is marginal as compared with multimodality treatment including transcatheter arterial embolization (TAE), hepatic arterial infusion chemotherapy (HAIC) or local ablation therapy [9]. However, huge HCC often presented with poor liver reserve, with increased frequency of intrahepatic metastasis and vascular invasion, which made surgical resection unsuitable. So transcatheter arterial embolization/chemoembolization (TAE/TACE) has been considered as the choice for the palliative treatment of huge unresectable HCG [10]. However, previous studies found that TACE for huge HCC had poor effect, and TACE related mortality rate of $6.5-20 \%$ were reported [10-11]. HAIC is another option for palliative treatment for inoperable advanced HCC [12-15]. From our study in 2004, HAIC with cisplatin, mitomycin G, leucovorin and 5-FU for advanced unresectable HCG has a tumor response rate of $28.3 \%$ and only one patient died due to the complication of HAIC during 211 courses of treatments [16]. From another recent study of our hospital, HAIC for advanced HCC had an overall response rate of $20 \%$ [17]. However, the effect of HAIC for the treatment of huge unresectable HCC remained unclear.

The aim of this study is to investigate the effect of HAIC versus symptomatic treatment for the treatment of huge unresectable HCG. 


\section{Materials and Methods}

\section{Patients}

From Janunary 2000 to December 2005, consecutive eligible patients with hepatocellular carcinoma (HCG) were enrolled in this study. HCC was diagnosed by pathology or elevation of alphafetoprotein (AFP) level above $400 \mathrm{ng} / \mathrm{ml}$ along with at least two different imaging techniques. All patients met the following criteria: (a) tumor of $8 \mathrm{~cm}$ or more in diameter, (b) patients who were not suitable for operation, (c) platelet counts $>50000 /$ cumm, (d) prothrombin time INR $<1.5$. (e) white cell counts $>2500 /$ cumm, and (f) Child A or B liver reserve. Patients with a previous history of treatment for HCG, or distant metastasis were excluded. Fifty-eight patients who received HAIC and 44 patients who received symptomatic treatment entered this study. In Taiwan, many patients did not like to receive chemotherapy, although they can fit the criteria for HAIC. Among the 44 patients who received symptomatic treatment, 16 patients had patent portal vein, but they refused TAE/TACE and another 28 patients who had thrombosis of portal vein refused HAIC.

\section{Ethics Statement}

The study was approved by the Kaohsiung Veterans General Hospital Institutional Review Board (VGHKS13-CT11-10). This was a retrospective study without intervention or obtaining clinical specimens and all the data were analyzed anonymously, so informed consent was waived. The waiving of informed consent was approved by the Institutional Review Board of Kaohsiung Veterans General Hospital.

\section{Hepatic Arterial Infusion Chemotherapy (HAIC)}

The left subclavian artery was cannulated with a catheter and the tip of the catheter was placed in the proper hepatic artery under fluoroscopic guidance before each course of chemotherapy [13]. The main trunk of the gastroduodenal artery was occluded by metallic coil routinely. Continuous infusion of 5000 units $(5 \mathrm{cc})$ heparin solution daily was filled in the catheter for preventing occlusion by thrombosis. Each course of treatment was for 5 days. Cisplatin $\left(10 \mathrm{mg} / \mathrm{m}^{2}\right)$ and mitomycin-C $\left(2 \mathrm{mg} / \mathrm{m}^{2}\right)$ were dissolved in $50 \mathrm{ml}$ isotonic sodium chloride solution which was infused for 20-30 minutes each time and continued for 5 days. In addition, $100 \mathrm{mg} / \mathrm{m}^{2}$ of 5-fluorouracil (5-FU), dissolved in $250 \mathrm{ml}$ of isotonic sodium chloride solution was administered for 24 hours using an infusion pump for 5 days. Leucovorin $\left(15 \mathrm{mg} / \mathrm{m}^{2}\right)$ was given daily to improve the efficacy of 5-FU during HAIC. The interval between 2 courses of treatment was 3 to 4 weeks. Each patient received at least one session of treatment. Three-phase computed tomography (CT) scan of liver was done after every 2 courses of treatment. The treatment was terminated when patients received 6 courses of treatment or until clinical conditions of the patients were not suitable for another course of HAIC.

\section{Follow-up}

All patients in the symptomatic treatment group and in the HAIC group who completed chemotherapy received follow-up with liver function test, AFP, sonography and/or CT scan every 3-6 months.

\section{Assessment of Response}

The modified Response Evaluation Criteria in Solid Tumors (RECIST) was used for assessment of tumor response defined as follows: complete response: no evidence of neoplastic disease; partial response: At least a $30 \%$ decrease in the sum of diameters of viable (enhancement in the arterial phase) target lesions, taking as reference the baseline sum of the diameters of target lesions; stable disease: any cases that do not qualify for either partial response or progressive disease; progressive disease: an increase of at least $20 \%$ in the sum of the diameters of viable (enhancing) target lesions, taking as reference the smallest sum of the diameters of viable (enhancing) target lesions recorded since treatment started.

\section{Statistical Analysis}

The data were expressed as mean+standard deviation. Categorical variables were compared with the $X^{2}$ test or Fisher's exact test when appropriate and continuous variables were compared with the Mann-Whitney test. Overall survival was estimated using the Kaplan -Meier method and the difference was determined by the log-rank test. Univariate and multivariate analysis with age $(>$ 65 vs $<65$ years), sex (male vs. female), vascular invasion, Child classification (A vs B), Okuda stage (I vs. II-III), Cancer of the Liver Italian Program (CLIP) score (1-3 vs. 4-6), Barcelona Clinic Liver Cancer (BCLC) stage (A-B vs. C-D), AFP level (>1000 vs < $1000 \mathrm{ng} / \mathrm{ml}$ ), and treatment (HAIC vs. symptomatic treatment) were performed using Cox's regression model with proportional hazards. A P-value of less than 0.05 was considered as statistically significant.

\section{Results}

The baseline characteristics of patients in the HAIC group and the symptomatic treatment group were similar in age, sex, tumor size, tumor number, the presence of main portal vein invasion, albumin level, bilirubin level, Child classification, Okuda stage, CLIP score, and BCLC stage (table 1).

A total of 134 courses of HAIC were performed for the 58 patients in the HAIC group. Each patient received 2.4+1.4 (range: 1-6) courses of HAIC. None of the patients died due to immediate HAIC complications. Adverse events of patients who received HAIC according to National Cancer Institute Common Terminology Criteria for Adverse Events (NCI-CTC AE) grading were described in Table 3. Eight patients developed fever during HAIC and among them, four patients developed bacteremia and were treated successfully by antibiotics. One patient developed overt subcutaneous hematoma at the puncture site but recovered soon and did not require further management. No vascular complications including occlusion or vasculitis of the hepatic artery were found.

Mean follow-up time was 10+11 months (range: 1-52 months). The median survival time is $9.5(1.5-50)$ months in the HAIC group and $3.0(1-45)$ months in the symptomatic treatment group $(\mathrm{P}=0.001)$. The overall survival rates at one and two years were $29 \%$ and $14 \%$ in the HAIC group and $7 \%$ and $5 \%$ in the symptomatic treatment group. The patients in the HAIC group had significantly better overall survival than those in the symptomatic treatment group $(\mathrm{P}<0.001)$ (Figure 1). Univariate and multivariate analysis of factors associated with mortality was shown in Table 2. Multivariate analysis revealed that the significant factors associated with the overall survival were treatment method (HAIC vs. symptomatic treatment, relative risk (RR): $0.321,95 \%$ confidence interval (CI): 0.200-0.515, P< 0.001), Child classification (A vs. B, RR: 0.537 , 95\% CI: $0.321-$ $0.901, \mathrm{P}=0.019)$ and CLIP score (1-3 vs 4-6, RR:0.61 1, 95\% CI: $0.375-0.997, \mathrm{P}=0.048)($ table 2$)$.

Tumor responses could be evaluated in 53 patients (39 in the HAIC group and 14 patients in the symptomatic treatment group) who survived for more than 3 months and had a measurable tumor on CT scan. In the HAIC group, 7 had responses (2 had 
Table 1. Baseline characteristics of the patients.

\begin{tabular}{|c|c|c|c|}
\hline & HAIC N= 58 & Symptomatic treatment $N=44$ & P-value \\
\hline Age (years) ${ }^{a}$ & $61 \pm 12$ & $63 \pm 17$ & .407 \\
\hline $\operatorname{Sex}(M / F)$ & $50 / 8$ & $32 / 12$ & .130 \\
\hline $\mathrm{HBV} /$ non-HBV & $35 / 23$ & $20 / 24$ & .135 \\
\hline Tumor size $(\mathrm{cm})^{a}$ & $11 \pm 3$ & $11 \pm 3$ & .277 \\
\hline Tumor No $(1 />1)$ & $25 / 33$ & $18 / 26$ & .824 \\
\hline Albumin (g/dl) ${ }^{a}$ & $3.3 \pm 0.6$ & $3.1 \pm 0.5$ & .177 \\
\hline Bilirubin (mg/dl) ${ }^{a}$ & $1.3 \pm 0.7$ & $1.5 \pm 0.8$ & .262 \\
\hline $\operatorname{AFP}(>1000 /<1000 \mathrm{ng} / \mathrm{ml})$ & $31 / 27$ & $24 / 20$ & 1.000 \\
\hline Main portal vein invasion $(+/-)$ & $32 / 26$ & $28 / 16$ & .390 \\
\hline Child class (A/B) & $30 / 28$ & $20 / 24$ & .530 \\
\hline Okuda stage(1/2-3) & $6 / 52$ & $5 / 39$ & 1.000 \\
\hline CLIP score(1-3/4-6) & $37 / 21$ & $23 / 21$ & .242 \\
\hline$B C L C$ stage $(A-B / C-D)$ & $16 / 42$ & $13 / 31$ & 1.000 \\
\hline
\end{tabular}

a Data presented as mean $+/-$ standard deviation.

AFP: alpha-fetoprotein, CLIP: Cancer of the Liver Italian Program, BCLC: Barcelona-Clinic Liver Cancer.

doi:10.1371/journal.pone.0092784.t001

complete responses and 5 had partial responses), 12 had stable diseases and 20 had progressive diseases. In the symptomatic treatment group, no patients had responses, 5 had stable diseases and 9 had progressive diseases. The rate of tumor response in measurable patients was higher in the HAIC group than the symptomatic treatment group but not statistically significant $(\mathrm{P}=0.176)$.

\section{Discussion}

Huge HCC is difficult to treat. Surgical resection is the treatment of choice for patients with huge $\mathrm{HCC}$ and wellpreserved liver function $[7,18]$. However, only a small proportion of patients with huge HCG can fit the criteria for surgical resection. But patients with huge $\mathrm{HCC}$ often had a higher prevalence of extracapsular tumor invasion into liver parenchyma, more frequent intrahepatic metastasis and worse survival than

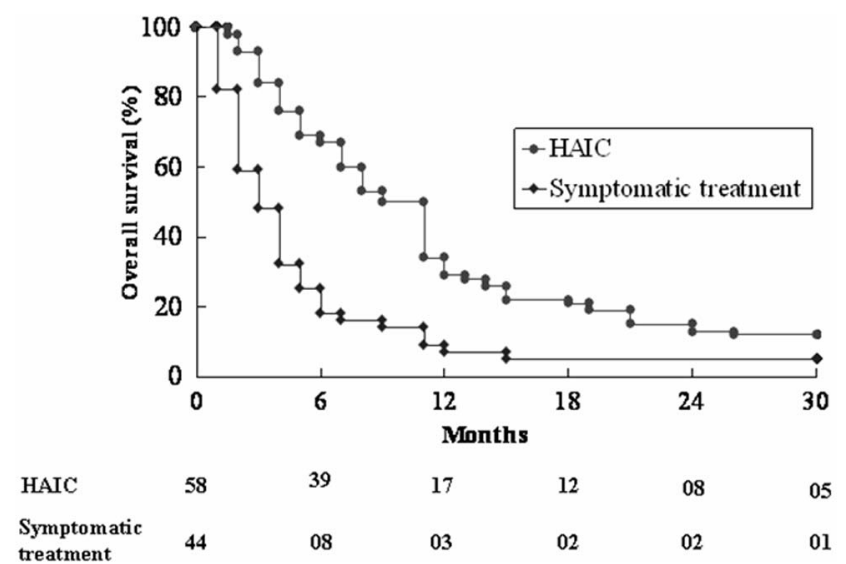

Figure 1. Comparison of the overall survival rate between the HAIC and symptomatic treatment group. The HAIC group had significantly better survival than the symptomatic treatment group $(\mathrm{P}<$ $0.001)$.

doi:10.1371/journal.pone.0092784.g001 those with smaller tumors [19-21]. There remained some controversies regarding the treatment for huge unresectable HCC.

During the 134 courses of HAIC, most patients tolerated the procedure well and no patients died of the immediate complications of HAIC. So HAIC may be a more safe treatment option for huge $\mathrm{HCC}$.

The overall response rate in the HAIC group was $18 \%$ but no patients in the symptomatic treatment group had response. Response was evaluated only in patients who survived for more than 3 months. Twenty-one out of 44 patients in the symptomatic treatment group survived for more than 3 months. The better survival in the HAIC group may be due to the higher response rate.

Patients with huge unresectable HCG were hard to treat and had poor prognosis. Two-year survival rate for patients with huge unresectable HCG who received symptomatic treatment was only $5 \%$ in this study. This finding was in accordance with the result of previous study by Huang et al [10], who found that the two-year survival rate for patients with huge unresectable HCC who received symptomatic treatment was $7.7 \%$. This study found that HAIC had survival benefit over symptomatic treatment for huge unresectable $\mathrm{HCC}$, and was also a safe treatment procedure. Therefore, we should encourage patients with huge HCC to receive HAIC to prolong survival if surgical resection was not suitable.

According to the study by Yamasaki et al [12], tumor size was not a prognostic factor that influenced the outcome of HAIC for patients with advanced HCC. But large tumor size was found to be associated with poor outcome of TACE for patients with HCC $[11,22,23]$. Although TAE/TACE has been considered as the palliative treatment for huge unresectable HCC in many institutes, the treatment outcome is generally poor [10-11], the survival benefit of TAE/TACE in these patients remained controversial and the mortality rate due to immediate complications of TAE/ TACE for huge HCG ranged from $6.5-20 \%$ in previous studies $[10,11]$. In our unpublished data that compared the outcome of $\mathrm{HAIC}$ and TAE for huge HCG, we found that the overall survival rates of the HAIC group was higher than the TAE group but did not reach statistical significance $(\mathrm{P}=0.077)$. Besides, $12 \%$ of patients in the TAE group died due to the immediate complica- 
Table 2. Factors associated with overall mortality in patients who receive HAIC or symptomatic treatment.

\begin{tabular}{|c|c|c|c|c|c|c|}
\hline & \multicolumn{3}{|c|}{ Univariate analysis } & \multicolumn{3}{|c|}{ Multivariate analysis } \\
\hline & Hazard ratio & $95 \% \mathrm{Cl}$ & $\mathbf{P}$ & Hazard ratio & $95 \% \mathrm{Cl}$ & $\mathbf{P}$ \\
\hline Age (>65 vs. $<65$ yrs) & 1.175 & $0.774-1.785$ & 0.449 & & & \\
\hline Sex (Male vs. female) & 1.113 & $0.655-1.890$ & 0.692 & & & \\
\hline Child's classification (A vs. B) & 0.430 & $0.279-0.662$ & $<0.001$ & 0.537 & $0.321-0.901$ & 0.019 \\
\hline PV thrombosis (yes vs. no) & 1.346 & $0.880-2.059$ & 0.171 & & & \\
\hline AFP level (>1000 vs $<1000 \mathrm{ng} / \mathrm{ml}$ ) & 0.683 & $0.450-1.036$ & 0.073 & & & \\
\hline Okuda stage (I vs. II-III) & 0.608 & $0.311-1.189$ & 0.146 & & & \\
\hline HAIC vs symptomatic treatment & 0.394 & $0.258-0.603$ & $<0.001$ & 0.321 & $0.200-0.515$ & $<0.001$ \\
\hline CLIP sore (1-3 vs. $4-6)$ & 0.482 & $0.315-0.736$ & 0.001 & 0.611 & $0.375-0.997$ & 0.048 \\
\hline BCLC stage (A-B vs. C-D) & 0.562 & $0.348-0.908$ & 0.018 & & & \\
\hline
\end{tabular}

HAIC: Hepatic arterial infusion chemotherapy, CLIP: Cancer of the Liver Italian Program, BCLC: Barcelona Clinic Liver Cancer.

doi:10.1371/journal.pone.0092784.t002

tions. In this study we found HAIC is safe and had survival benefit over symptomatic treatment for huge unresectable HCG. Further randomized controlled study to compare the treatment outcome of HAIC versus TAE/TACE for huge unresectable HCG is required. Besides, main portal vein thrombosis was found in 60 out of 102 patients with huge unresectable HCG in this study. Main portal vein thrombosis was a contraindication for TAE/ TACE but not a contraindication for HAIC. So HAIC should be considered as an important treatment option for huge unresectable HCC especially when main portal vein thrombosis was present.

Multivariate analysis found that HAIC, Child classification and CLIP score were significantly associated with better survival rate. Child classification has been found to be associated with the outcome of patients with HCG treated with HAIC, TACE [1213] or local ablation therapy [24] in our study. CLIP score also was found to be associated with the outcome of patients with HCC treated with TAGE [25-26]. Both liver reserve and tumor stage remained to be the important prognostic factors in patients with huge unresectable HCC.

Sorafenib has been developed and is recommended for the treatment of advanced HCC [27,28]. But the effect of sorafanib for HCG is not satisfactory and actually the response rate of sorafenib is low. In the Asia-Pacific study by Cheng et al, the overall survival was 6.5 months in patients treated with sorafenib, compared with 4.2 months in those who received placebo and only $3.3 \%$ of patients achieved a partial response and no patients had complete response [29]. Effects of sorafenib in patients with huge unresectable $\mathrm{HCC}$ is unclear. Besides, sorafenib is limited by a high cost and many patients can not afford to receive the treatment. The treatment outcome of TACE for patients with huge unresectable HCC is generally poor [10-11]. In countries where sorafenib is not available or too expensive for patients to afford, HAIC is a good treatment option.

HAIC has been found to have benefits in HCG patients with portal vein thrombosis (PVT) [30,31]. Recently Lau et al reported that transarterial radioembolization (TARE) is a good treatment option in HCC patients with PVT [32]. In patients with unresectable HCG who have developed PVT, TARE may prolong survival with minimal impact on quality of life. Further studies are required to compare the effects of HAIC vs. TARE in patients of HCG with PVT.

This study has several limitations. This is not a randomized controlled study, and selection bias may be possible in this study. But the baseline characteristics including age, sex, liver reserve, tumor stages are similar between the two groups of patients and after multivariate analysis, the HAIC group still has survival benefit over patients who received symptomatic treatment. In a recent non-randomized study from Japan [30], Nouso et al. found HAIC has survival benefit over symptomatic treatment in advanced HCG. This is in accordance with the result of our study. A randomized controlled study to compare the treatment outcome of HAIC versus symptomatic treatment for huge

Table 3. Adverse events of patients who received HAIC according to $\mathrm{NCI}-\mathrm{CTC}$ AE grading.

\begin{tabular}{lll}
\hline Clinical toxicities & Grade I-II, n (\%) & Grade III-IV, n (\%) \\
\hline Leukopenia & $7(12)$ & $2(3.4)$ \\
Anemia & $3(5.2)$ & $0(0)$ \\
Thrombocytopenia & $4(6.9)$ & $0(0)$ \\
Elevated aminotransferase & $8(13.8)$ & $3(5)$ \\
Hyperbilirubinemia & $8(13.8)$ & $0(0)$ \\
Elevated creatinine & $2(3.4)$ & $0(0)$ \\
Nausae/vomiting & $25(43)$ & $0(0)$ \\
Diarrhea & $5(8.6)$ & $0(0)$ \\
Fever & $4(6.9)$ & $4(6.9)$ \\
\hline doi:10.1371/journal.pone.0092784.t003 & &
\end{tabular}


unresectable HCG has not been reported before and it is not ethical to place patients untreated during randomization.

In conclusion, HAIC is a safe procedure and provides better survival than symptomatic treatment for patients with huge unresectable HCG.

\section{References}

1. Kao JH, Chen DS (2005) Changing disease burden of hepatocellular carcinoma in the FarEast and Southeast Asia. Liver Intern 25: 696-703.

2. El-Serag HB, Mason AC (1999)Rising incidence of hepatocellular carcinoma in the United States. N Engl J Med 340: 745-50.

3. Sheu JC, Sung JL, Chen DS, Lai MY, Wang TH, et al. (1985)Early detection of hepatocellular carcinoma by real-time ultrasonography. A prospective study. Cancer 56: 660-6.

4. Llovet JM, Schwartz M, Mazzaferro V (2005) Resection and liver transplantation for hepatocellular carcinoma. Sem Liver Disease 25: 181-200

5. Bruix J, Sherman M, Llovet JM, Beaugrand M, Lencioni R, et al. (2001) EASL panel of experts on HCC: clinical management of hepatocellualr carcinoma. Conclusions of the Barcelona-2000 EASL conference. European Association for the Study of the Liver. J Hepatol 35: 421-30.

6. NoguchiT KawaradaY, KitagawaM ItoF, SakuraiH, et al. (1997) Clinicopathologic factors influencing the long-term prognosis following hepatic resection for large hepatocellular carcinoma more than $10 \mathrm{~cm}$ in diameter. Semin Oncol (Suppl. 6): 7-13.

7. Lee NH, Chau GY, Lui WY, King KL, Tsay SH, et al. (1998) Surgical treatment and outcome in patients with a hepatocellular carcinoma greater than $10 \mathrm{~cm}$ in diameter. Br J Surg 85: 1654-7.

8. Furuta T, Sonoda T, Matsumata T, Kanematsu T, Sugimachi K, et al. (1992) Hepatic resection for a hepatocellular carcinoma larger than $10 \mathrm{~cm}$. J Surg Oncol 51: 114-7.

9. Mok KT, Wang BW, Lo GH, Liang HL, Liu SI, et al. (2003) Multimodality management of hepatocellular carcinoma larger than $10 \mathrm{~cm}$. Journal of the American College of Surgeons 197: 730-8.

10. Huang YH, Wu JC, Chen SC, Chen CH, Chiang JH, et al. (2006) Survival benefit of transcatheter artherial cheomoembolization in patients with Hepatocellular carcinoma larger than $10 \mathrm{~cm}$ in diameter. Alim Pharm Therp 23: $129-35$.

11. Poon RTP, Ngan H, Lo CM, Liu CL, Fan ST, et al. (2000) Transarterial chemoembolization for inoperable Hepatocellular carcinoma and postresection intrahepatic recurrence. J Surg Oncol 73: 109-14.

12. Yamasaki T, Kimura T, Kurokawa F, Aoyama K, Ishikawa T, et al. (2005) Prognostic factors in patients with advanced Hepatocellular carcinoma receiving hepatic arterial infusion chemotherapy. J Gastroenterol 40: 70-8.

13. Ando E, Tanaka M, Yamashita F, Kuromatsu R, Yutani S, et al. (2002) Hepatic arterial infusion chemotherapy for advanced hepatocellular carcinoma with portal vein tumor thrombosis: analysis of 48 cases. Cancer 95: 588-95.

14. Chung YH, Song H, Song BC, Lee GC, Koh MS, et al. (2000) Combined therapy consisting of intraarterial cisplatin infusion and systemic interferonalpha for hepatocellular carcinoma patient $5 \mathrm{~s}$ with major portal vein thrombosis or dist ant metastasis. Cancer 88: 1986-91.

15. Urbe T, Kaneko S, Matsusuta E, Unoura M, Kobayashi K, et al. (1998). Clinical pilot study of intrahepatic arterial chemotherapy with methotrexate, 5fluouracil, cisplatin and subcutaneous interferon-alpha-2b for patients with locally advanced hepatocellular carcinoma. Oncology 55: 39-47.

16. Lin CP, Yu HC, Cheng JS, Lai KH, Lo GH, et al, (2004) Clinical effects of intra-arterial infusion chemotherapy with cisplatin, mitomycin $\mathrm{C}$, leucovorin and 5-fluorouracil for unresectable advanced Hepatocellular carcinoma. J Chin Med Assoc 67: 602-10.

\section{Author Contributions}

Conceived and designed the experiments: WLT KHL JSC. Performed the experiments: WLT KHL JSC PIH HLL HHC WCG HCY FWT HMW. Analyzed the data: WLT KHL JSC HLL HCT. Contributed reagents/ materials/analysis tools: WLT KHL JSG HLL HCT. Wrote the paper: WLT.

17. Liang HL, Huang JS, Lin YH, Lai KH, Yang CF, et al. (2007) Hepatic arterial infusion chemotherapy for advanced hepatocellular carcinoma by placing a temporary catheter via the subclavian route. Acta Radiologica 48: 734-40.

18. Poon RT, Fan ST, Wong J (2002) Selection criteria for hepatic resection in patients with large hepatocellular carcinoma larger than $10 \mathrm{~cm}$ in diameter. J AM Coll Surg 194: 592-602.

19. Hsu HC, Sheu JC, Lin YH, Chen DS, Lee CS, et al, (1985) Prognostic histologic features of resected small hepatocellular carcinoma (HCC) in Taiwan- a comparison with resected large HCC. Cancer 56: 672-80.

20. Jwo SC, Chiu JH, Chau GY, Loong CC, Lui WY, et al. (1992) Risk factors linked to tumor recurrence of human hepatocellular carcinoma after hepatic resection. Hepatology 16: 1367-71.

21. Adachi E, Maeda T, Kajivama K, Kinukawa N, Matsumata T, et al. (1996) Factors correlated with portal venous invasion by hepatocellular carcinoma: univariate and multivartiate analyses of 232 resected cases without preoperative treatment. Cancer 77: 2022-31.

22. Takayasu K, Arii S, Ikai I, Omata M, Okita K, et al. (2006) Prospective cohort study of transarterial chemoembolization for unresectable hepatocellular carcinoma in 8510 patients. Gastroenterol 131: 461-9.

23. Yoshioka H, Sato M, Sonomura T, Terada M, Kishi K, et al. (1997) Factors associated with survival exceeding 5 years after transcatheter arterial embolization for hepatocellular carcinoma. Semin Oncol 24;suppl 6: S29-37.

24. Tsai WL, Cheng JS, Lai KH, Lin CP, Lo GH, et al. (2008) Percutaneous acetic acid injection versus percutaneous ethanol injection for small hepatocellular carcinoma : a long-term follow-up study. Alim Pharmacol Ther 28: 304-11.

25. The Cancer of the Liver Italian Program (CLIP) investigators (1998) A new prognostic system for patients with hepatocellular carcinoma: a retrospective study of 435 patients. Hepatology 28: 751-5.

26. Huang YH, Chen $\mathrm{CH}$, Chang TT, Chen SC, Chiang JH, et al. (2005) The role of transcatheter arterial embolization for patients with unresectable hepatocellular carcinoma: a nationawide, multi-center study evaluated by cancer stages. Alim Pharmacol Ther 21: 687-94.

27. Bruix J, Sherman M (2001) Management of hepatocellular carcinoma: an update. Hepatology 53: 1020-22.

28. El-Serag HB, Hepatocellular carcinoma (2011) N Engl J Med 365: 1118-27.

29. Cheng AL, Kang YK, Chen Z,Tsao GJ, Qin S, et al. (2009) Efficacy and safety of sorafenib in patients in the Asia-Pacific region with advanced hepatocellular carcinoma: a phase III randomised, double-blind, placebo-controlled trial. Lancet Oncol 10(1): 25-34.

30. Nouso K, Miyahara K, Uchida D, Kuwaki K, Izumi N, et al. (2013) Effect of hepatic arterial infusion chemotherapy of 5-fluorouracil and cisplatin for advanced hepatocellular carcinoma in the Nationwide Survey of Primary Liver Cancer in Japan. Br J Cancer 109: 1904-7.

31. Song do S, Bae SH, Song MJ, Lee SW, Kim HY, et al. (2013) Hepatic arterial infusion chemotherapy in hepatocellular carcinoma with portal vein tumor thrombosis. World J Gastroenterol 19: 4679-88.

32. Lau WY, Sangro B, Chen PJ, Cheng SQ, Chow P, et al. (2013) Poon RT Treatment for hepatocellular carcinoma with portal vein tumor thrombosis: the emerging role for radioembolization using yttrium-90. Oncology 84(5): 311-8. 